\title{
Patterns of Zooplankton Functional Response in Communities with Vertical Heterogeneity: a Model Study
}

\author{
A. Morozov ${ }^{a b 1}$, E. Arashkevich ${ }^{b}$ \\ ${ }^{a}$ Department of Mathematics, University of Leicester, Leicester, LE1 7RH, UK \\ ${ }^{b}$ P.P. Shirshov Institute of Oceanology, RAS, 36 Nakhimovskiy Prospekt, \\ Moscow, 117997, Russia
}

\begin{abstract}
Parameterization of zooplankton functional response is crucial for constructing plankton models. Theoretical studies predict enhancing of system stability in case the response is of sigmoid type. Experiments on feeding in laboratories tell us in favor of non-sigmoid types for most herbivorous zooplankton species. However, recent field observations show that the overall functional response of zooplankton in the whole euphotic zone can exhibit a sigmoid behavior even when the response for the same species in laboratory mesocosms is non-sigmoid. Here we propose a simple model explaining the observed alterations of functional response. We divide the euphotic zone into a number of layers and take into account the food-dependent migration of zooplankton. In each layer, the functional response (local response) is suggested to be non-sigmoid. We show that the overall response of zooplankton exhibits different behavior compared to the patterns of the local response. In particular, the model predicts emergence of a sigmoid type as a result of zooplankton accumulation and feeding in layers with high phytoplankton density. We show the importance of light attenuation by phytoplankton on the alteration of functional response. The modelling results allow us to hypothesize that the sigmoid functional response in real communities should emerge more often than it was suggested earlier based only on experimental studies on zooplankton feeding.
\end{abstract}

Keywords: sigmoid functional response; conceptual plankton models; Holling's types; vertical distribution

AMS subject classification: 92B05, 92D25, 92D40

\footnotetext{
${ }^{1}$ Corresponding author. E-mail: am379@le.ac.uk
} 


\section{Introduction}

Mathematical models of plankton dynamics are of great interest because they improve our understanding of basic mechanisms controlling functioning of plankton communities. Earlier plankton models were based on the classical 'mean-field' approach. According to this approach, an ecosystem is described at population scale and the spatial distribution of species is ignored. Such simplification often leads to contradictions with reality. For instance, the mean-field approach predicts population cycles of large amplitude in ecosystems with high nutrient concentration. This is often at odds with observations [23]. A great deal of model artifacts disappears when we take into account spatial heterogeneity of plankton [29, 34].

The importance of space in plankton models is now well recognized. Sophisticated 3D spatial models have been constructed taking into account particularities of hydrological regimes in areas under study. Those models usually contain a large number of variables with the aim to provide a detailed description of the ecosystem. Note, however, that construction and implementation of complicated spatially explicit models have some serious drawbacks as well. The point is that we often lose tractability of models and such an approach does not provide us with a clear understanding of factors controlling system dynamics. We can only compute numerical solution for a few sets of parameters while the whole bifurcation structure remains unknown. An important question is: whether we can simplify spatial multi-species models to make them more tractable, however, avoiding the shortcomings that we had with the earlier 'mean-field' models?

One possible alternative is the use of the 'modified mean-field' approach [27, 28]. This approach suggests changing parameter values in the right-hand side functions of the earlier meanfield equations (e.g. growth rate, predation rate, etc.) to incorporate implicitly effects of spatial aggregation and clustering of species. The modified mean-field approach was successively applied in different fields of theoretical ecology $[13,27]$. Another way to incorporate implicitly the space is the use of 'the scale transition approach' based on the moment approximation method [5, 8].This technique allows to extrapolate results obtained with small-scale experiments to scales of natural habitats. Such an extrapolation can be done by adding into the initial mean-field equations the so-called moment closure terms. Finally, incorporating of space implicitly can be done via the aggregation approach $[2,18]$. The aggregation approach is especially helpful in case we model an ecosystem with pronounced heterogeneity of physical environment, when the whole system can be divided into a few connected habitats with different living conditions. Under certain conditions, the dynamics of such network of habitats can be described by a simplified model in terms of mean species densities [3, 24, 31].

In this paper, we apply the ideas of the aggregation approach to reveal patterns of zooplankton feeding in plankton communities with non-homogeneous vertical species distribution. In those communities, the pronounced heterogeneity of physical environment in the vertical direction is caused by light attenuation and stratification processes [32]. Note that zooplankton can perform selective feeding in layers with high food supply $[15,16,22,36]$. We are interested in behavior of functional response of herbivorous zooplankton in the euphotic zone as a function of total amount of food.

The zooplankton functional response is defined as the food intake rate (per biomass of a zoo- 
plankter per unit of time) as a function of phytoplankton density $P$. Conventionally, patterns of zooplankton response are classified into three categories known as Holling types $[12,17]$. These are: Holling type I (a direct proportionality between the intake rate and P); Holling type II (concave downward with a further saturation); Holling type III (sigmoid response). Note as well that the functional response can depend as well on the amount of zooplankton in the water column $[10,22,30]$.

The choice of type of zooplankton response is of great importance for constructing plankton models [11]. It is well known that the sigmoid type enables system stability at low algal densities which is problematic with non-sigmoid types $[4,26]$. Also, the use of Holling type III would decrease amplitudes of oscillations of phytoplankton-zooplankton cycles [34]. Some models (e.g. 'red tide' models) simply do not 'work' when we replace Holling type III with Holling type II or I [35].

The conventional way to determine the type of response for a given zooplankton species consists in laboratory experiments. Those experiments are usually carried out in small containers or tanks where the algal concentration is artificially varied and the rate of food consumption is measured. In most cases, Holling types I and II have been obtained for herbivorous zooplankton $[19,20]$. This fact has made some researchers rather skeptical concerning the use of Holling type III in plankton modelling [6, 34].

Recently, it was shown [25] that the overall functional zooplankton response (i.e. the consumption rate of the entire zooplankton population in the water column as a function of total amount of food in real ecosystems) can exhibit a qualitatively different behavior compared to patterns observed in laboratory experiments for the same species. In particular, a sigmoid type of response for the whole population can emerge even when the response of individual zooplankters is nonsigmoid. The apparent alteration was suggested to occur because of the ability of selective feeding of zooplankton in layers with high algal density. Note that some recent experiments in plankton towers also tell us in favor of the above explanation [22].

In this paper, we suggest a simple theoretical framework explaining the observed alteration of functional response. We split the euphotic zone into a number of layers and consider the fooddependant exchange of zooplankton between the layers. Also, we take into account the light attenuation by algae of upper layers. The local feeding of zooplankton is described by non-sigmoid functions (Holling types I or II). We are interested in the shape of the overall response (obtained by averaging of local responses over the layers) as a function of total amount of food. Note that this is exactly the overall functional response which is implied in mean-field plankton models. We obtained that the overall response for the whole zooplankton population shows a qualitatively different behavior compared to that of the local response. We found that a sigmoid response can emerge in the ecosystem as a result of interplay between selective zooplankton grazing and selfshading of light by phytoplankton. The appearance of sigmoid response is facilitated with an increase of the total amount of zooplankton in the system. We discuss the conditions which are necessary to obtain an overall sigmoid response from local non-sigmoid ones according to the suggested mechanism. 


\section{Modelling framework}

We split the whole euphotic zone into a number of horizontal layers. In this paper, we consider only two layers; however, such an approach allows an extension of the model to an arbitrary number of layers. In layer $i$, variations of densities of zooplankton $\left(Z_{i}\right)$ and phytoplankton $\left(P_{i}\right)$ are described by the following ordinary differential equations:

$$
\begin{aligned}
& \frac{d P_{i}}{d t}=\tilde{r}_{i} P_{i}-f\left(P_{i}\right) Z_{i}, \\
& \frac{d Z_{i}}{d t}=G_{i}\left(Z_{i}, P_{i}\right)+\Lambda_{i},
\end{aligned}
$$

where $i=1,2$ correspond to the upper layer and to the lower layer, respectively; $\tilde{r}_{i}$ is the phytoplankton growth rate, $f$ is the zooplankton consumption rate ('local' functional response); the term $\Lambda_{i}$ describes the exchange of zooplankton between the layers due to vertical migration; the function $G_{i}\left(Z_{i}, P_{i}\right)$ stands for variation in zooplankton density due to mortality and/or predation by carnivorous zooplankton, birth processes and increase of biomass due to feeding. We neglect active vertical migration of phytoplankton. For the sake of simplicity, we consider that the layers have the same width.

In this paper, we consider that the whole biomass of zooplankton and, hence the average zooplankton density, vary on a slower time scale compared to the rate of phytoplankton multiplication and the rate of exchange of zooplankton between the layers. This signifies that $G_{1}\left(Z_{1}, P_{1}\right) \approx$ $G_{2}\left(Z_{2}, P_{2}\right) \approx 0$. For the average zooplankton density in the water column we have:

$$
\frac{d\left(Z_{1} / 2+Z_{2} / 2\right)}{d t}=0
$$

Thus, on the considered fast time scale, the average zooplankton density becomes a model parameter, which we denote by: $Z_{0}=\left(Z_{1}+Z_{2}\right) / 2$. Such an assumption is based on the fact that the characteristic time scale for phytoplankton growth is approximately $0.5-1$ day (see, for example, [15]. The characteristic time scale of changing of shape of vertical zooplankton profile (due to vertical displacements of zooplankters) is close to this value. Note, however, that the rate of vertical displacement of an individual zooplankter may be higher. At the same time, the characteristic time scale of zooplankton multiplication is of order of 1-2 weeks. From this point of view, our modelling framework is different from the aggregation approach applied in [24, 31], where migration rates of species were considered to be much faster than their growth rates, allowing to consider the density of all species in the system as slow variables.

To proceed further, we need to parameterize the right-hand side functions of equations (2.12.2). The rate $\Lambda_{i}$ of exchange of zooplankton between the layers is due to two main processes: (i) active displacement of zooplankton from a layer with poorer food conditions to the one with better food conditions (food taxis) and (ii) exchange of zooplankton between layers due to the Brownian diffusion. This can be described by:

$$
\Lambda_{i}=\tilde{\Lambda}_{i}+D\left(Z_{j}-Z_{i}\right)
$$


where $\tilde{\Lambda}_{i}$ stands for the food taxis and $D\left(Z_{j}-Z_{i}\right)$ stands for the Brownian diffusion.

We parameterize the food taxis of zooplankton as being proportional to the difference of food concentration between the layers. This is called a density-dependent migration [2]:

$$
\tilde{\Lambda}_{1}=\left\{\begin{array}{ll}
D_{1} Z_{2}\left(P_{1}-P_{2}\right), & P_{1}>P_{2} \\
D_{1} Z_{1}\left(P_{1}-P_{2}\right), & P_{1}<P_{2}
\end{array} \quad, \quad \tilde{\Lambda}_{2}=-\tilde{\Lambda}_{1}\right.
$$

The condition $\tilde{\Lambda}_{1}=-\tilde{\Lambda}_{2}$ is due to the equal widths of the layers; $D_{1}$ and $D_{2}$ are positive coefficients. To parameterize the phytoplankton growth rate we use a logistic function, i.e., $\tilde{r}_{i} P_{i}=r_{i} P_{i}\left(1-P_{i} / K\right)$ where $K$ is the carrying capacity, $r_{1}$ and $r_{2}$ are constants. However, we take into account attenuation of growth of phytoplankton in the lower layer by the phytoplankton from the upper layer, when the abundance of $P_{1}$ is high. We describe the self-shading via a standard way [15] by multiplying $r_{2}$ by $\exp \left(-\gamma P_{1}\right)$, i.e., we suggest that the light attenuation coefficient is proportional to phytoplankton density. We arrive at the following system of four ordinary differential equations for the plankton densities in the upper layer 1 and the lower layer 2 :

$$
\begin{gathered}
\frac{d P_{1}}{d t}=r_{1} P_{1}\left(1-P_{1} / K\right)-f\left(P_{1}\right) Z_{1}, \\
\frac{d Z_{1}}{d t}=\tilde{\Lambda}_{1}+D\left(Z_{2}-Z_{1}\right), \\
\frac{d P_{2}}{d t}=\exp \left(-\gamma P_{1}\right) r_{2} P_{2}\left(1-P_{2} / K\right)-f\left(P_{2}\right) Z_{2}, \\
\frac{d Z_{1}}{d t}=-\tilde{\Lambda}_{1}+D\left(Z_{1}-Z_{2}\right),
\end{gathered}
$$

where $\tilde{\Lambda}_{1}$ is given by (2.5). In this paper, we consider two different parameterizations of local response $f$ :

$$
\begin{gathered}
f\left(P_{i}\right)=\alpha P_{i} \\
f\left(P_{i}\right)=\frac{\alpha P_{i}}{1+\beta P_{i}}
\end{gathered}
$$

These are Holling type I and Holling type II functional responses, respectively $[12,17]$. In this paper, we are interested in the behaviour of the overall functional response of zooplankton which is obtained by averaging the local responses over the whole water column:

$$
F=\frac{f\left(P_{1}\right) Z_{1}+f\left(P_{2}\right) Z_{2}}{Z_{1}+Z_{2}}
$$

This value is to be plotted versus the space average density of phytoplankton, i.e., versus $P=$ $\left(P_{1}+P_{2}\right) / 2$.

The use of (2.12) needs some comments. First of all, this expression might be non-constant in case $P_{i}(t)$ and $Z_{i}(t)$ oscillate. However, this difficulty can be easily settled. In case of oscillations 
(periodic or chaotic), the overall functional response should be understood as the time average $<$ $F>$ over the system trajectories. This value is to be plotted versus the time average $\langle P\rangle$. For the sake of convenience, we omit the symbols $<>$ further on. Second, the overall functional response $F$ might not be a function of the average phytoplankton density $P$ in a strict mathematical sense, since the same value of $\mathrm{P}$ might correspond to different sets of $\left(P_{1}, P_{2}\right)$. We call this phenomenon a multiple functional response. The possibility of a multiple functional response is one of serious drawbacks of mean-field modelling. We shall address this issue in more details in Discussion section. Here, by agreement, we plot all values of consumption rate $F$ for a given $P$ that we obtain in the model.

Finally, to reveal the dependence of $F$ on $P$, we need a certain continuous range of $P$, since for any fixed set of model parameters we may obtain only finite set of $P$, corresponding to different system attractors. For example, if the system has only one stationary state (or limit cycle), we have, obviously, only a single value of $P$ which tells nothing about the behaviour of $F$. To obtain a continuous range of $P$, we vary model parameters. We assume that the parameters describing local feeding of zooplankton and zooplankton migration behaviour remain constant and we change only the parameters describing the rate of phytoplankton multiplication. We apply the following algorithm. We fix the average zooplankton density and plot $F$ versus $P$ by progressively increasing $r_{i}$. To reveal the dependence of the overall functional response on the total amount zooplankton in the system, we repeat the same procedure for different $Z_{0}$. Conceptually, this algorithm is close to the one applied by [1]. Note also that implementation of such a technique will allow us to model the scenario typically observed in practice when the growth rate of phytoplankton increases (or decreases) mostly due to changes in sun luminosity or temperature. As a result, a phytoplankton bloom can be triggered depending on functional type of functional response of zooplankton [35].

We use $\mu g \mathrm{Cl}^{-1}$ as units of phyto- and zooplankton densities. We consider the system parameters to vary within the following ranges: $0.05<r_{i}<2$ 1/day [7]; $25<K<100 \mu \mathrm{g} \mathrm{Cl}^{-1}$ [9]; $0.01<\alpha<0.21 /\left(\mu g C l^{-1}\right.$ day); $0.005<\beta<0.11 / \mu g C l^{-1}[14,33]$. The attenuation constant can be estimated from [16]. We consider, approximately, that the total depth of the water column is 75-100 m. This gives us an estimate for : $0.05<\gamma<11 /\left(\mu g C l^{-1}\right)$. Estimating the values of $D$ and $D_{1}$ is a quite complicated issue. In this paper, we consider $0.25<D_{1}<21$ /day and $0.5<D<51 /\left(\mu g C l^{-1}\right.$ day). This allows us to obtain biologically reasonable values of $P$ and $Z$ with the other parameters varying within the indicated ranges. Overall, we emphasize that here we are interested in the qualitative behavior of the system and the exact values of parameters are not of first importance.

\section{Results}

First, we consider the situation when the local functional response of zooplankton is given by the linear function (2.10). For the sake simplicity, we focus mainly on the case when $r_{1}=r_{2}=r$ , i.e. the attenuation of algal growth in the lower layer is mostly due to the light shading by the phytoplankton of the upper layer. However, we shall briefly discuss the case when $r_{1}>r_{2}$ as well. 
In Appendix, we analyze the stationary states of model (2.6-2.9) with (2.10) and $r_{1}=r_{2}$. It is shown that the phytoplankton cannot persist in the system if its growth rate is less than $r=\alpha Z_{0}$. Thus we increase the value of $r$ starting from $\alpha Z_{0}$. Note that for any fixed $r$, the system has only one attractor. This is either a stable stationary state or a limit stable cycle.

The behavior of the overall functional response $F$ depends on the total amount of zooplankton in the system which is characterized by $Z_{0}$. Fig. 1 shows $F$ constructed for different $Z_{0}$. The figure is obtained by numerical integration of (2.6-2.9) over large periods of time ( $T>100$ days). We also plot the proportions of phyto- and zooplankton dwelling in the upper layer, i.e., the quantities $\eta_{P}=\left(P_{1}+P_{2}\right) / 2$ and $\eta_{Z}=\left(Z_{1}+Z_{2}\right) / 2$. By solid line we show the functional response $(\alpha P)$ that we would have for a fully homogeneous system $\left(P_{1}=P_{2} ; Z_{1}=Z_{2}\right)$ for the same average phytoplankton densities.

Typical behavior of $F(P)$ for small $Z_{0}$ is represented in Fig.1a $\left(Z_{0}=0.2 \mu g \mathrm{Cl}^{-1}\right)$. At low phytoplankton density (i.e. for $r \approx \alpha Z_{0}$ ), the overall functional response is linear and close to $\alpha P$. This is due to the fact that the plankton is equally distributed between the layers. At high phytoplankton densities, the response becomes close to a linear one again. This is due the fact that almost all plankton is concentrated in the upper layer $\left(\eta_{P} \approx \eta_{Z} \approx 1\right)$. However, the slope of $F$ is now twice of that what we have at low $P$. This can be easily seen from:

$$
F=\frac{\alpha P_{1} Z_{1}+\alpha P_{2} Z_{2}}{Z_{1}+Z_{2}} \approx \alpha P_{1} \approx 2 \alpha P
$$

The increase of the slope from $\alpha$ to $2 \alpha$ signifies that there exists a range of $P$ such that the response of the whole system increases more rapidly than linearly. Note that theoretical studies would predict enhancing of stability of an ecosystem with a functional response having such a property $[4,26]$.

For a larger amount of zooplankton in the system, the behavior of functional response becomes sigmoid (see Fig. 1b, $Z_{0}=3 \mu g C l^{-1}$ ). At low $P$, the response is close to the linear one; however, for larger $P, F(P)$ exhibits a concave upward behaviour. After passing the inflection point, $F(P)$ becomes concave downward and approaches $2 \alpha P$. Thus, accumulation and feeding of zooplankton in the layer with higher phytoplankton density would result in the emergence of a Holling type III response for non-sigmoid local responses.

For a further increase of $Z_{0}$, another interesting phenomenon takes place (Fig. 1c, $Z_{0}=5 \mu \mathrm{gCl} \mathrm{l}^{-1}$ and Fig. $\left.1 \mathrm{~d}, Z_{0}=10 \mu \mathrm{g} C l^{-1}\right)$. Within a certain range of $P$, the same average phytoplankton density corresponds to three different consumption rates (i.e. we have a multiple functional response). For higher $P$, the overall functional response becomes again a function of $P$ and can be approximated by $2 \alpha P$. Here the multiple functional responses are not related to a hysteresis behavior (e.g. the existence of multiple stationary states), since for any fixed $r$, the system is characterized by a unique set $\left(P_{1}, P_{2}\right)$ (see Appendix). We will call the range of $P$ corresponding to multi-functional response the $\mathrm{S}$-shaped region.

The appearance of the S-shaped region can be explained as follows. With an increase in $r$, the proportion of phytoplankton in the lower layer decreases due to inhibition by $P_{1}$. After the density $P_{1}$ attains some critical range, the growth of phytoplankton in the lower layer becomes severely depressed and $P_{2}$ exhibits a sharp decline. As a result, the average density of phytoplankton in 

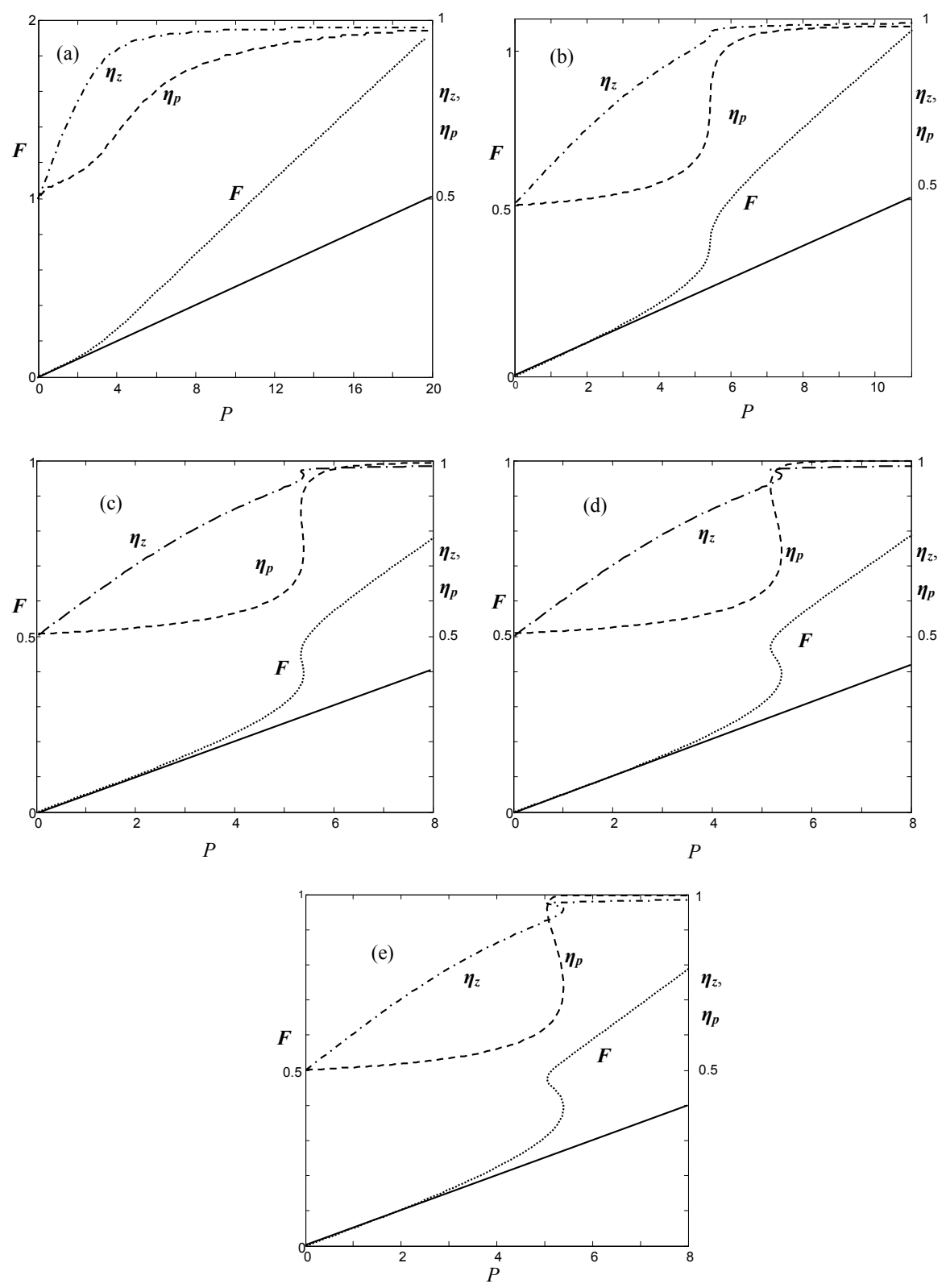

Figure 1: The overall functional response $F$ (1/day) constructed for different total amounts for zooplankton $Z_{0}$ in the system. The local functional responses are linear; the phytoplankton growth rates are equal: $r_{1}=r_{2}$. The functions $\eta_{P}$ and $\eta_{Z}$ represent relative proportion of phytoplankton and zooplankton in the upper layer. The model parameters are the following: $\alpha=0.051 /\left(\mu \mathrm{gCl} \mathrm{l}^{-1}\right.$ day); $K=50 \mu g C l^{-1} ; \gamma=0.41 /\left(\mu g C l^{-1}\right) ; D_{1}=0.51 /$ day; $D=21 /\left(\mu C l^{-1}\right.$ day). (a) $Z_{0}=0.2 \mu g C l^{-1}$; (b) $Z_{0}=3 \mu g C l^{-1}$; (c) $Z_{0}=5 \mu g C l^{-1}$; (d) $Z_{0}=10 \mu g C l^{-1}$; (e) $Z_{0}=20 \mu g C l^{-1}$. Solid line shows the functional response $(\alpha P)$ which would take place in the homogeneous system. 
the whole water column becomes smaller. With a further increase of $r$, the value of continues to increase and this increase compensates the loss of phytoplankton in the lower layer and the average density of phytoplankton increases again. This increase is monotone until $P_{1}=K$. The location of the S-shaped region can be characterized by the point $P_{0}$ which is defined by:

$$
P_{0}=\frac{P_{\max }+P_{\min }}{2}
$$

where $P_{\max }$ and $P_{\min }$ are the local maximum and minimum of $P$ when we consider $P$ as a function of $F$ in the S-shaped region. A further increase in $Z_{0}$ does not lead to any qualitative change in behavior of functional response (Fig. 1e, $Z_{0}=20 \mu \mathrm{C} \mathrm{Cl}^{-1}$ ). Note that possibility of multiple functional responses is one of drawbacks of models with implicit space and we discuss possible solution of this problem in Section 4.

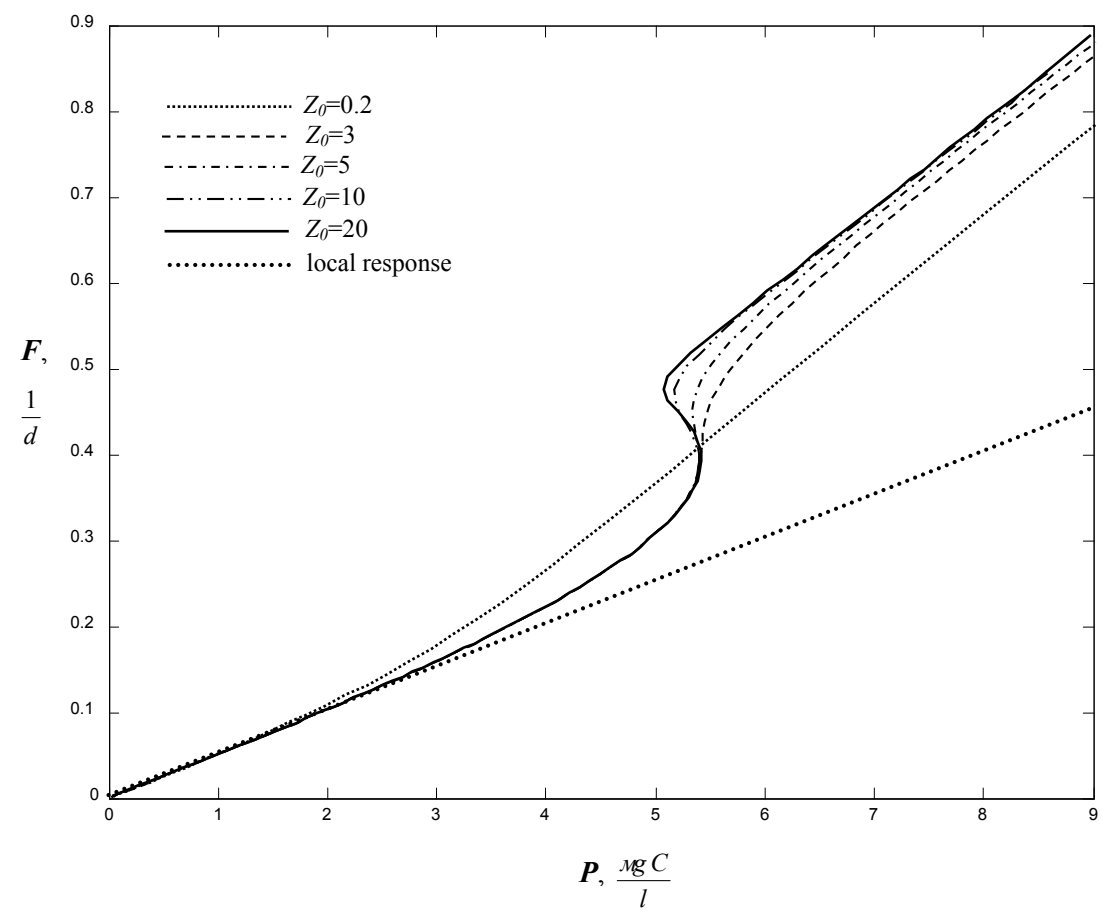

Figure 2: The overall functional response $F$ obtained for different amount of zooplankton (see fig.1). With an increase in $Z_{0}, F$ approaches the curve represented by a solid line.

To understand better how the total amount of zooplankton affects the overall response, we plot $F(P)$ in the same graph for different $Z_{0}$ (see Fig.2). We show the local functional response as well. One can see that the strength of overall response depends on $Z_{0}$; however, pronounced alteration of the shape of $F$ is observed only for small $Z_{0}$. For large $Z_{0}$, the shape of $F$ becomes independent of total amount of zooplankton and approaches the curve which is shown by a solid line. The functional response depends only on food density. Note that transformation of the functional response with increasing of predator density in (2.6-2.9) has a different underlying mechanism compared to 


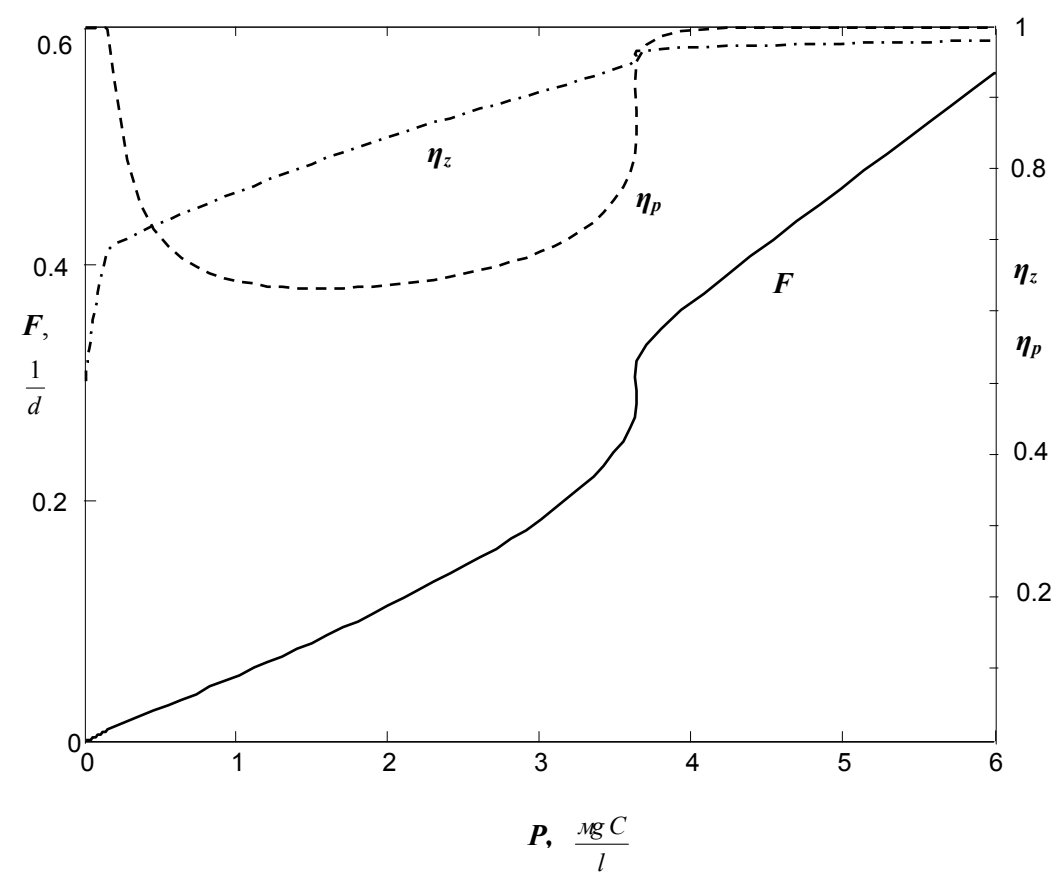

Figure 3: The overall functional response $F$ constructed in the case when local functional responses are linear and different phytoplankton growth rates $r_{1}=\omega r_{2}, \omega=0.5 ; \eta_{P}$ and $\eta_{Z}$ represent the relative proportions of phytoplankton and zooplankton in the upper layer. Here $Z_{0}=10 \mu g C l^{-1}$; the other model parameters are the same as in fig. 1.

a direct interference of predators reported in ecological literature (see Discussion). The behavior of $F(P)$ shown in fig.1,2 remains qualitatively the same for others sets of the system parameters.

Let us consider briefly a more realistic situation when the algal growth in the lower layer is inferior to that of the upper layer at small $P$ (due to a strong absorption of light by the water), i.e., we consider that $r_{2}=\omega r_{1}$, where $\omega<1$. The behavior of $F$ becomes more complicated. For instance, there can be more than one intervals of $P$ with concave downward $F$. The new interval with concave downward behavior is observed in the vicinity of the origin and for narrow parameter range. A detailed bifurcation diagram should be constructed somewhere else. We should say that the basic results obtained for remain. Fig. 3(constructed numerically) shows the functional response plotted for $r_{2}=0.5 r_{1}, Z_{0}=10 \mu \mathrm{gCl} \mathrm{l}^{-1}$. The persistence of phytoplankton in the system becomes possible for $r_{1}>\alpha Z_{0}$. At very low $P$, the overall functional response is given by $2 \alpha P$. This is due to the fact that phytoplankton in the lower layer cannot persist; hence the whole consumption takes place only in the upper layer. With an increase in $r_{1}$, the growth of $P_{1}$ becomes possible, $\eta_{P}$ deviates from 1 . The light attenuation by $P_{1}$ is not yet large enough to depress the growth of $P_{2}$. The relative proportions of plankton in both layers are close to each other. The overall response can be approximated by $\alpha P$. With an increase in $P$, the overall response shows a sigmoid behavior which is similar to the one obtained for $r_{2}=r_{1}$. Finally, the response approaches again $2 \alpha P$, i.e. the same slope as at small $P$. Note that the position of $\mathrm{S}$-shaped region becomes 
shifted towards the origin. This fact can be easily understood since the amount of $P_{1}$, which is necessary to cause a decline of the growth of $P_{2}$, is now smaller because $r_{2}<r_{1}$.

An important question is how the above results will change when the local functional response $f$ is non-linear. Here we considered the situation when $f$ is of Holling type II parameterized by function (2.11), which is most often used in plankton literature for parametrization of this functional type. We should say that the bifurcation structure of the model in case of hyperbolic local response becomes much more complicated and a detailed investigation should be done somewhere else. For example, we found that an alternation of concave downward and concave upward behavior can take place several times. Here we provide a brief insight into the influence of saturation of local response on patterns of the overall response.

It is convenient to use the value of $P_{0}$ (which characterizes the position of the S-region for the model with linear local response) for predicting the behavior of the system with the hyperbolic local response. For $P_{0} \ll 1 / \beta$, (i.e. the half-saturation of local response occurs at algal densities much higher than $P_{0}$ ) the emergence of a sigmoid response is possible (see fig.4a, $\beta=0.02 \mu \mathrm{g} C \mathrm{l}^{-1}$ ). Note that the overall response for large $P$ becomes of Holling type II. In case $P_{0} \approx 1 / \beta$, the saturation of local response starts playing an important role (see fig. $4 \mathrm{~b}$, $\left.\beta=0.1 \mu g C l^{-1}\right)$. Numerical analysis shows that there exists a small $\mathrm{S}$-shape region as well as some ranges of $P$ where the functional response is concave downwards. However, the conclusion that the overall response should be described by a sigmoid function in this case would be rather questionable. Note that in both cases in fig.4, the rate of overall consumption of phytoplankton by zooplankton is higher than in the case of homogeneous vertical distribution (shown by a solid line). Finally, for $P_{0} \gg 1 / \beta$, the overall functional response becomes close to the local one (2.11) due to saturation effects and no sigmoid response is possible. Thus, an important condition for emergence of sigmoid response in model (2.6-2.9) is $P_{0} \ll 1 / \beta$, where $P_{0}$ is defined by (3.2).

\section{Discussion}

Construction of plankton models requires a rather careful choice of parametrization of zooplankton functional response. Recent observations show that the heterogeneity of vertical distribution of plankton as well as active displacement of zooplankters can alter patterns of zooplankton feeding $[22,25]$. In this paper, we suggest a simple modelling framework to explain the observed alterations. Another important aspect of the study is the necessity of understanding what kind of patterns of overall response we would find in a plankton community for a given local functional response and a given migration strategy of zooplankton. This would be of importance for constructing mean-field models.

The model shows a surprising discrepancy between the behaviour of overall response in a vertically distributed plankton community and in the community where the organisms are well mixed. In particular, we demonstrate that a sigmoid functional response can emerge in case when the local responses are non-sigmoid. This observation is of practical interest because the sigmoid functional response would enhance stability of the ecosystem $[4,26]$. Note that alteration of shape 

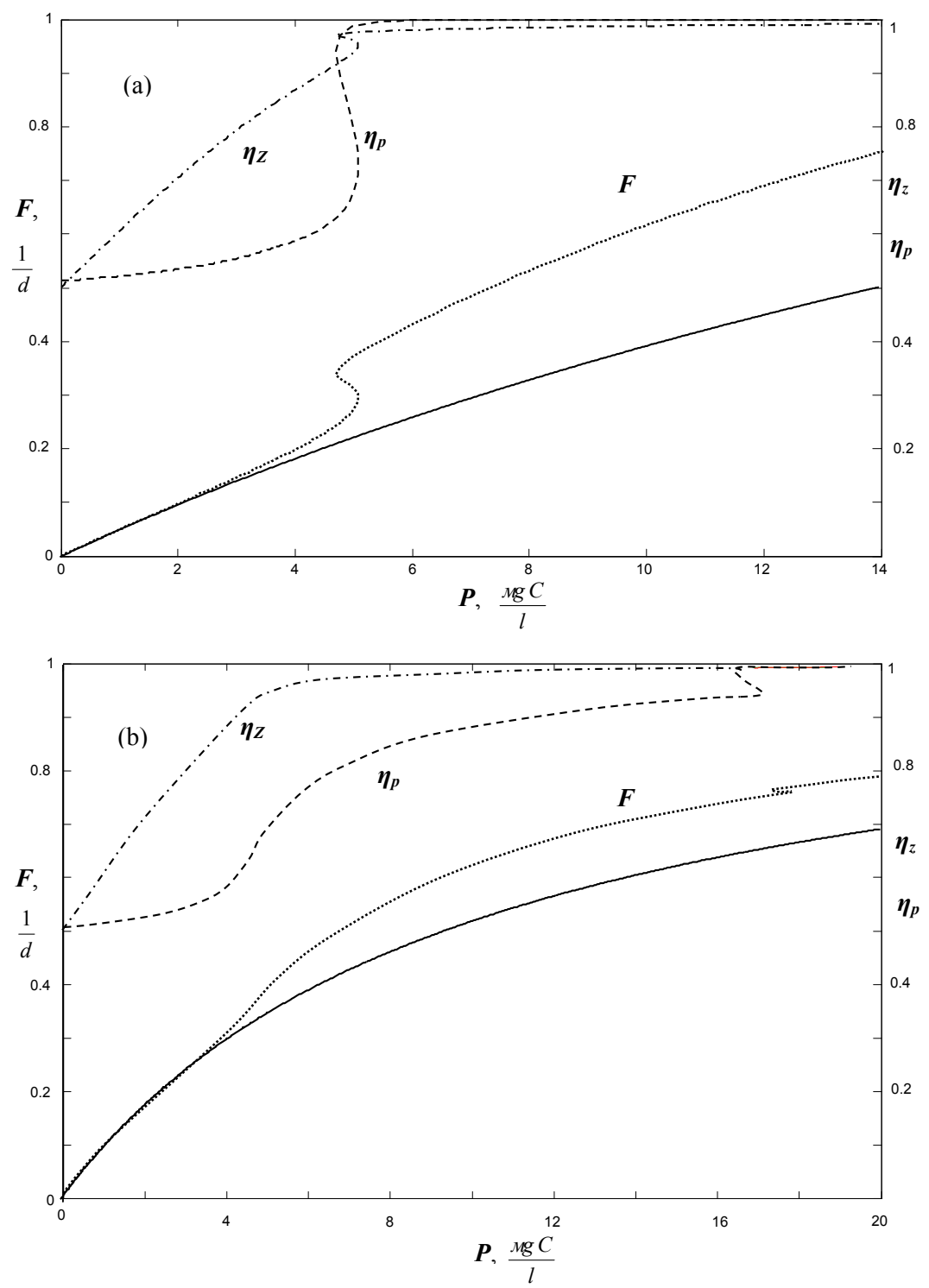

Figure 4: The overall functional response $F$ in case when local functional responses are of Holling type II. The functions $\eta_{P}$ and $\eta_{Z}$ represent the relative proportions of phytoplankton and zooplankton in the upper layer. (a) The half-saturation density is low: $1 / \beta \gg P_{0}\left(\beta=0.021 / \mu g C l^{-1}\right)$; (b) the half-saturation density is high: $1 / \beta \cong P_{0} \quad\left(\beta=0.1 \quad 1 / \mu g C l^{-1}\right)$, where $P_{0}$ is defined by (3.2). Here $r_{1}=r_{2} ; Z_{0}=10 \mu g C l^{-1}$, the other model parameters are the same as in fig.1. In both cases the solid line shows the functional response (2.11) which would take place in the homogeneous system. 
functional response, while going from a small laboratory scale to scales of the whole habitat, has been reported for some other ecosystems [7, 37]. An important particularity of our study is that such an alteration modifies not only the parameters but also the type of response.

Our results seem to be of importance, since a large number of real communities are characterized by a pronounced vertical heterogeneity and active vertical movement of zooplankton. A fundamental issue is whether the conventional method of parametrization of functional response is correct. In other words, a direct incorporation of a response obtained in laboratory experiments into mean-field modelling can be erroneous. In particular, the fact that non-sigmoid types are reported in experiments for most herbivorous zooplankton species $[6,20]$ does not signify automatically that we shall not observe a Holling III for those species in real ecosystems.

We found that the emergence of Holling type III in a system with local responses of Holling type I and II requires the following conditions: (i) heterogeneity of spatial distribution of food which becomes more and more pronounced as the total amount of food increases; (ii) active displacement (migration) of zooplankton and its selective feeding in layer(s) with high food density. The light attenuation is one of key feature of the suggested mechanism because it enhances the gradient of food distribution while the total amount of food is increasing. As a result, the proportion of zooplankton migrating to layers with better food conditions will increase with an increase of total amount of food. We should note also that to have a pronounced sigmoid response, the total amount of zooplankton in the system should be sufficiently high (cf. fig.2). Finally, fast saturation of local functional responses can impede the emergence of sigmoid response (see fig.4).

The sigmoid functional response in our model has different underlying mechanism compared to the one reported earlier in ecological literature. The point is that, conventionally, the concave upward part of Holling III response was suggested to occur at low food density. This is due to alteration of hunting behavior of zooplankton and/or switching to another food source when the main source becomes depleted [12] and the references therein). In our model, the functional response at low food density is linear (provided the local responses are of Holling type I or II). This is due to homogeneous spatial distribution of food. The concave upward part emerges at intermediate food density as a result of displacement of zooplankton to layers with higher food density and not due to altering in hunting behavior. The inflection point of $F$ in (2.6-2.9) is a consequence of alteration of functioning of the whole ecosystem, when the interplay between the grazing of zooplankton and the light attenuation leads to an abrupt decline of phytoplankton in the lower layer and almost all zooplankton becomes concentrated in the upper layer.

The overall functional response in (2.6-2.9) can be described as a function of average density of phytoplankton $P$; however, the model predicts also the existence of 'multiple functional responses' when the same $P$ corresponds to different consumption rates $F$. This should not be considered as a model artifact. Indeed, field observations often show that ecosystems with close values of total biomass of phytoplankton exhibit rather different vertical distributions. As a result, the total consumption rates in those systems would show a pronounced difference. Note the possibility of such a situation is one of major difficulties of implementation of the mean-field approach. However, in case when the multiple functional response arises only within a small range of phytoplankton density (see fig.1), it is possible to settle the mentioned problem. We can construct an overall functional response $\tilde{F}(P)$ which is close to $F$ (different criteria of closeness can be chosen) but 
which would be a unique function of $P$. Further, the function $\tilde{F}(P)$ can be used to describe the functional response in a mean-field model approximating the initial multi-layer system. Note that the construction of $\tilde{F}(P)$ close to the non-unique function $F$ should be a matter of a separated paper. Here, our main concern was demonstrating scenarios of alteration of functional response emerging in the system.

The model predicts that the overall functional response would depend on the total amount of predator in the system (see fig.2). Such kind of dependence is a topic of debates in ecological literature with different mechanisms suggested. In plankton models, the predator dependence can emerge as intratrophic predation [30]. Another important mechanism is the direct competition between zooplankton species. It is known that some species release chemicals to reduce the consumption rate of algae by their competitors [10]. Our model explains the predator-dependence in a different way. With an increase of the total amount of zooplankton, the spatial distribution of food becomes more heterogeneous due to intensive grazing. This results in a more effective selective feeding of zooplankton in layers with high food density. Note also that the dependence of $F$ on $Z_{0}$ (see Fig.2) is rather different compared to the well known patterns in the literature. In particular, the functional response in model (2.6-2.9) becomes independent of $P$ at high values of $Z_{0}$.

Our study leave some questions open. An important issue is a possible influence of diel vertical migration of zooplankton on the patterns of feeding. It is well known that some zooplankton species stay in the upper layer and graze on phytoplankton only at night and descend during daytime into deep layers with poor food $[21,36]$. This might require a different modelling approach by considering daily-based forcing of the system. Another important issue is an active vertical displacement of some phytoplankton by modifying their buoyancy [32]. Thus, high percentage of phytoplankton in the upper layer, as a result of an increase in $P$, can occur due to ascending of algae from deeper layers and not because of high mortality of algae of deep layers. Finally, it is important to take into account the non-heterogeneous vertical distribution of limiting nutrients in the euphotic zone. Due to consumption of nutrients by algae, the upper layer becomes more depleted in nutrients than the lower one and the maximum of phytoplankton growth rate can occur in the lower layer. Definitely, the influence of the above factors on the overall functional response should be investigated. However, we should say also that the model may lose its tractability in this case.

Finally, we would like to emphasize that a very little attention has been paid so far in the literature to reveal patterns of zooplankton response in real ecosystems. This is rather in contrast with a huge amount of laboratory studies on the experimental feeding of zooplankton. Thus, one objectives of this paper is to urge plankton researchers to fill the gap between the extensive research in the field of experimental feeding in tanks and the scares results on feeding patterns in reality.

\section{Appendix}

Here we analyze the stationary states of system (2.6-2.9) with local functional response given by (2.10). We consider the case $r_{1}=r_{2}=r$. 
The system has the trivial stationary state $\left(0,0, Z_{0}, Z_{0}\right)$ with phytoplankton densities in both layers equal to zero. By using the standard technique of stability analysis it is easy to show that the trivial state is stable if and only if $r-\alpha Z_{0}<0$. In other words, the phytoplankton is 'overgrazed' in case its growth rate is less than $\alpha Z_{0}$.

There are two stationary states characterized by zero density of phytoplankton in only one layer. They are given by: $\left(0, \tilde{P}_{2}, Z_{1}, Z_{2}\right)$ and $\left(\tilde{P}_{1}, 0, Z_{1}, Z_{2}\right)$.

Let us consider first the stationary state $\left(0, \tilde{P}_{2}, Z_{1}, Z_{2}\right)$, where the value of $\tilde{P}_{2}$ is determined from the following quadratic equation:

$$
\tilde{P}_{2}\left(2 Z_{0}-\left(1-\frac{\tilde{P}_{2}}{K}\right) \frac{r}{\alpha}\right)-\frac{D_{1}}{D}\left(2 \frac{r}{\alpha}\left(1-\frac{\tilde{P}_{2}}{K}\right)-2 Z_{0}\right)=0
$$

The stationary zooplankton densities are defined by:

$$
Z_{1}=\left(1-\frac{\tilde{P}_{2}}{K}\right) \frac{r}{\alpha}, \quad Z_{2}=2 Z_{0}-Z_{1}
$$

By considering (4.1), one can easily prove that the $\left(0, \tilde{P}_{2}, Z_{1}, Z_{2}\right)$ exists if and only if $r-\alpha Z_{0}>$ 0 . This stationary state is always unstable.

For the stationary state $\left(\tilde{P}_{1}, 0, Z_{1}, Z_{2}\right)$ we have similar equation for $\tilde{P}_{1}$ :

$$
\tilde{P}_{1}\left(2 Z_{0}-\left(1-\frac{\tilde{P}_{1}}{K}\right) \frac{r}{\alpha}\right)-\frac{D_{1}}{D}\left(2 \frac{r}{\alpha}\left(1-\frac{\tilde{P}_{1}}{K}\right)-2 Z_{0}\right)=0
$$

with $Z_{1}=r\left(1-\tilde{P}_{1} / K\right) / \alpha$ and $Z_{2}=2 Z_{0}-Z_{1}$.

By using the standard technique of stability analysis, one can prove that $\left(\tilde{P}_{1}, 0, Z_{1}, Z_{2}\right)$ is unstable in the case:

$$
r \exp \left(-\gamma \tilde{P}_{1}\right)>2 Z_{0} \alpha-\left(1-\tilde{P}_{1} / K\right) r
$$

The system also possesses a nontrivial stationary state $\left(P_{1}^{*}, P_{2}^{*}, Z_{1}^{*}, Z_{2}^{*}\right)$. The value of $P_{1}^{*}$ can be determined from the following transcendental equation:

$$
P_{1}^{*}-P_{2}^{*}\left(P_{1}^{*}\right)-\frac{D}{D_{1}}\left(\frac{1-P_{1}^{*} / K}{2 \alpha Z_{0} / r-1+P_{1}^{*} / K}-1\right)=0
$$

where $P_{2}^{*}\left(P_{1}^{*}\right)$ is given by:

$$
\left.P_{2}^{*}\left(P_{1}^{*}\right)=\left(\left(-2 \alpha Z_{0} / r+1-P_{1}^{*} / K\right) \exp \left(\gamma P_{1}^{*}\right)+1\right)\right) K
$$

The stationary densities of zooplankton can be found from:

$$
Z_{1}^{*}=\left(1-P_{1}^{*} / K\right) r / \alpha, \quad Z_{2}^{*}=2 Z_{0}-Z_{1}^{*}
$$


The analytical treatment of (4.6) and (4.7) shows that in case when $r-\alpha Z_{0}>0$ holds and, at the same time, condition (4.4) has the opposite sign, the system possesses a nontrivial stationary state and only one. In this case, the state $\left(\tilde{P}_{1}, 0, Z_{1}, Z_{2}\right)$ is unstable. The trivial stationary state is also unstable. When (4.4) is satisfied, the system does not have the nontrivial stationary state and the state $\left(\tilde{P}_{1}, 0, Z_{1}, Z_{2}\right)$ is stable. The loss of stability of $\left(\tilde{P}_{1}, 0, Z_{1}, Z_{2}\right)$, as a result of variation of parameters, takes place via a transcritical bifurcation, when this state collides with the non-trivial stationary state.

The nontrivial stationary state $\left(P_{1}^{*}, P_{2}^{*}, Z_{1}^{*}, Z_{2}^{*}\right)$ can be stable or unstable, depending on system parameters. Numerical simulations show that the loss of stability of the state takes place via a supercritical Hopf bifurcation with a birth of small stable limit cycle. Note that the stability loss takes place for large values of $Z_{0}$.

\section{Acknowledgements}

We highly appreciated S.V. Petrovskii for a careful reading and comments.

\section{References}

[1] R. Arditi,Y. Tyutyunov, A Morgulis, V. Govorukhin, I. Senina. Directed movement of predators and emergence of density-dependence in predator-prey models. Theor. Pop. Biol., 59 (2001), 207-221.

[2] P. Auger, J.C. Poggiale, S. Charles. Emergence of individual Behaviour at the population level: effects of density dependent migration on population dynamics. Compte Rendus de l'Acadmie des Sciences, Sciences de la Vie, 323 (2000), 119-127.

[3] P. Auger, S. Charles, M. Viala, J.C. Poggiale. Aggregation and emergence in ecological modelling: integration of ecological levels. Ecol. Mod., 127 (2000), 11-20.

[4] A.D. Bazykin. Nonlinear dynamics of interacting populations. Series on nonlinear science, Chua, L.O. (ed), Series A, 11. World Scientific, 1998.

[5] U. Bergstrum, G. Englund, K., Leonardsson. Plugging space into predator-prey models: an empirical approach. Am. Nat., 167 (2006), 246-259.

[6] W.R. DeMott. Feeding selectivities and relative ingestion rates of Daphnia and Bosmina. Limnol. Oceanogr., 27 (1982), 518-527.

[7] A.M. Edwards, J. Brindley. Zooplankton mortality and the dynamical behavior of plankton population models. Bull. Math. Biol., 61 (1999), 202-339.

[8] G. Englund, K. Leonardsson. Scaling up the functional response for spatially heterogeneous systems. Ecol. Lett., 11 (2008), 440-449. 
[9] P. Franks. Phytoplankton blooms in a fluctuating environment: the roles of plankton response time scales and grazing. J. Plankt. Res., 23 (2001), 1433-1441.

[10] C. Folt, C.R. Goldman. Allelopathy between zooplankton: a mechanism for interference competition. Science, 213 (1981), 1133 - 1135.

[11] G.F. Fussmann, B. Blasius. Community response to enrichment is highly sensitive to model structure. Biol. Lett., 1 (2005), 9-12.

[12] W. Gentleman, A. Leising, B. Frost, S. Storm, J. Murray. Functional responses for zooplankton feeding on multiple resources: a review of assumptions and biological dynamics. Deep-Sea Res. II, 50 (2003), 2847-2875.

[13] S. Gubbins, C.A. Gilligan. A test of heterogeneous mixing as a mechanism for ecological persistence in a disturbed environment. Proc. R. Soc. B., 264 (1997), 227-232.

[14] P.J. Hansen, P.K. Bjrnsen,B.W. Hansen. Zooplankton grazing and growth: scaling within the 2-2,000-m body size range. Limnol. Oceanogr., 42 (1997), 687-704.

[15] A.W. Herman. Vertical distribution patterns of copepods, chlorophyll, and production in northeastern Baffin Bay. Limnol. Oceanogr., 28 (1983), 709-719.

[16] A.W. Herman, T. Platt. Numerical Modelling of Diel Carbon Production and Zooplankton Grazing on the Scotian Shelf Based on Observational Data. Ecol. Mod., 18 (1983), 55-72.

[17] H.S. Holling. Some characteristics of simple types of predation and parasitism. Canad. Entomol., 91 (1959), 385-398.

[18] Y. Iwasa, V. Endreasen, S.A. Levin. Aggregation in model ecosystems I. Perfect aggregation. Ecol. Model. 37 (1987), 287-302.

[19] J.M. Jeschke, M. Kopp, R. Tollrian. Predator functional responses: discriminating between handling and digesting prey. Ecol. Mongr., 72 (2002), 95-112.

[20] J.M. Jeschke, M. Kopp, R. Tollrian. Consumer-food systems: why type I functional responses are exclusive to filter feeders. Biol. Rev., 79 (2004), 337-349.

[21] W. Lampert. Zooplankton vertical migrations: implications for phytoplankton-zooplankton interactions. Arch. Hydrobiol. Beih. Ergebn. Limnol., 35 (1992),69-78.

[22] W. Lampert. Vertical distribution of zooplankton: density dependence and evidence for an ideal free distribution with costs. BMC Biology, 3 (2005), 10 (electronic).

[23] E. McCauley, W.W. Murdoch. Predator-prey dynamics in environments rich and poor in nutrients. Nature, 343 (1990), 455-457.

[24] J. Michalski, J.C. Poggiale, R. Arditi,P. Auger. Macroscopic dynamic effects of migrations in patchy predator-prey systems. J. Theor. Biol., 185 (1997), 459-474. 
[25] A.Y. Morozov, E.G. Arashkevich, M. Reigstad, S. Falk-Petersen. Influence of spatial heterogeneity on the type of zooplankton functional response: a study based on field observations. Deep-Sea Res. II, (2008) in press.

[26] A. Oaten, W.W. Murdoch. Functional response and stability in predator-prey systems. Am. Nat., 109 (1975), 289-298.

[27] M. Pascual, M. Roy, A. Franc. Simple models for ecological systems with complex spatial patterns. Ecol Lett., 5 (2002), 412-419.

[28] M. Pascual. Computational ecology: From the Complex to the Simple and Back. PLoS Comput Biol., 1 (2005), 2 (electronic).

[29] S.V. Petrovskii, B.L. Li, H. Malchow. Transition to chaos can resolve the paradox of enrichment. Ecol. Complex., 1 (2004), 37-47.

[30] J. Pitchford, J. Brindley. Intrafunctional predation in simple predator-prey models. Bull. Math. Biol., 60 (1998), 937-953.

[31] J.C. Poggiale. Predator-Prey Models in Heterogeneous Environment: Emergence of Functional Response. Math. Comp. Model., 27 (1998), 63-71.

[32] J.E.G. Raymont. Plankton and Productivity in the Oceans.Phytoplankton. Pergamon, Oxford, 1980.

[33] E. Saiz, A. Calbet. Scaling of feeding in marine calanoid copepods. Limnol. Oceanogr., 52 (2007), 668-675.

[34] M. Scheffer, R.J. De Boer. Implications of spatial heterogeneity for the paradox of enrichment. Ecology, 76 (1996), 2270-2277.

[35] J.E. Truscott, J. Brindley. Ocean Plankton Populations as Excitable Media. Bull. Math. Biol., 56 (1994), 981-998.

[36] M.E. Vinogradov. Vertical Distribution of the Oceanic Zooplankton. Israeli Program for Scientific Translations, Jerusalem, 1970.

[37] F.M. Wilhelm, D.W. Schindler, A.S. McNaught. The influence of experimental scale on estimating the predation rate of Gammarus lacustris (Crustacea: Amphipoda) on Daphnia in an alpine lake. J. Plankton Res., 22 (2000), 1719-1734. 